\title{
The exemplar methodology: An approach to studying the leading edge of development
}

\author{
Kendall Cotton Bronk
}

\author{
*Correspondence: kcbronk@bsu.edu \\ Teachers College, 511 Department \\ of Educational Psychology, Ball \\ State University, 2000 West \\ University Avenue, Muncie, IN \\ 47306, USA
}

\begin{abstract}
The exemplar methodology is a useful, but to date underutilized, approach to studying developmental phenomena. It features a unique sample selection approach whereby individuals, entities, or programs that exemplify the construct of interest in a highly developed manner form the study sample. Studying a sample of highly developed individuals yields an important view of the leading edge of development that cannot be gleaned using other methodologies. A picture of the full range of development requires not only an understanding of typical and deficient growth, as provided by existing methodologies, but also of complete or nearly complete development, as provided by the exemplar methodology. Accordingly, the exemplar methodology represents a critical tool for developmental psychologists. In spite of this, because it has rarely been written about, the exemplar methodology has only been used to study a relatively narrow range of developmental constructs. Therefore, the present article defines the exemplar methodology, addresses key conceptual issues, and briefly outlines steps to utilizing the approach.
\end{abstract}

Keywords: Qualitative research, Qualitative methodology, Exemplar methodology, Moral development, Positive psychology, Positive youth development

A wide range of methods exists for studying developmental constructs, and each has its own strengths and weaknesses, but historically no methodology has existed that could yield a picture of the leading edge of development, predict the next steps in development for typical individuals, or feature instances of complete- or nearly complete- development of a particular characteristic, and without this view, a complete understanding of construct development is impossible (Damon \& Colby in press). The ability to view the upper ends of development in practice represents the unique contribution of the exemplar methodology.

The exemplar methodology is a sample selection technique that involves the intentional selection of individuals, groups, or entities that exemplify the construct of interest in a highly developed manner. This definition is derived from the empirical studies in developmental psychology that have employed this approach. In using the exemplar methodology, researchers deliberately select a sample of individuals or entities that exhibit a particular characteristic in a highly developed manner. The exemplar methodology features participants who are rare, not from the perspective of the characteristics they exhibit, but in the intensity with which they demonstrate those particular characteristics. For example, most people demonstrate care and compassion at times and with some level of sincerity, but care exemplars exhibit this characteristic more consistently and more intensely then more typical individuals. While exemplars differ from more typical individuals in terms of the

(c) 2012 Bronk; licensee Springer. This is an Open Access article distributed under the terms of the Creative Commons Attribution License (http://creativecommons.org/licenses/by/2.0), which permits unrestricted use, distribution, and reproduction in any medium, provided the original work is properly cited. 
way they exhibit a particular characteristic, they tend to be similar to more typical individuals in most other ways (Colby \& Damon 1992). For instance, a creative genius could be considered an exemplar, but just because this individual boasts particularly creative ideas does not mean that he or she is necessarily kinder, more sociable, or more athletic than other individuals. Exemplars serve as highly developed examples of the construct of interest, but in other ways their development may be typical or even deficient.

Studying individuals who exemplify different elements of development in highly developed ways is crucial; only by doing so are we able to witness advanced development in the real world. Exemplars trace the steps of where more typical individuals are likely to go, if growth continues (Colby \& Damon 1992). We call the individuals who exhibit a particular characteristic in an intense manner exemplars, and we call the methodology used to study these individuals the exemplar methodology.

\section{History of the exemplar methodology}

Although developmental psychologists interested in positive psychology have only relatively recently begun using the exemplar methodology (e.g. Colby \& Damon 1992), the methodology has been in existence since Aristotle. In Nicomachean Ethics Aristotle wrote, "We approach the subject of practical wisdom by studying the persons to whom we attribute it" (Aristotle 1962, 6.5 1140a25). In other words, to understand how a complex construct functions and develops, it is useful to examine that construct in the lives of individuals who exhibit it in a highly developed manner. Along these same lines, Maslow (1971) was one of the earliest scholars to actually employ the exemplar methodology, though he never called it that. Education, he claimed, "is learning to grow and learning what to grow toward" (p. 169). If we want to learn about ultimate human potential, he argued, we should study highly functional and enlightened individuals.

Use of the exemplar methodology has increased in the past twenty years in conjunction with the growth of the positive psychology movement (Seligman \& Csikszentmihalyi 2000; Sheldon \& King 2001; Damon 2004). Historically, psychologists were primarily concerned with understanding what could go wrong with regards to human behavior, emotions, social interactions, and cognition. The focus of this one-sided, however important, field of human functioning has brought about a highly developed understanding of people's mental vulnerabilities, deficiencies, and illnesses. However, this focus has essentially ignored issues of human thriving and flourishing (Benson et al. 2006; Bundick et al. 2010; Lerner et al. 2003; Seligman 2011). Leaders in the field of psychology, recognizing the need for additional knowledge of, research into, and practical methods to sustain people's inner strengths and overall well-being, helped establish the new paradigm of positive psychology.

As the number of studies based on this new paradigm has increased, so too has use of the exemplar methodology (e.g. Matsuba et al. in press). The exemplar methodology lends itself to the study of optimal human development. Using a methodology that is focused on the upper ends of development is appropriate in an area concerned with ideal states of being.

\section{Conceptual issues}

The exemplar methodology represents a complex approach to studying human development, and as such, there are many issues to consider when applying it. First, the 
decision of whether to include a comparison sample can be contentious. Many effective exemplar studies have included matched samples (e.g. Bronk 2008; Hart \& Fegley 1995; Matsuba \& Walker 2004; Matsuba \& Walker 2005; Reimer 2003; Walker \& Frimer 2007), but other valuable studies have not (e.g. Bronk 2011; Bronk 2012; Colby \& Damon 1992; Mastain 2008). Including a comparison sample allows the researcher to draw conclusions regarding ways in which the exemplar sample is distinct from more typical individuals, and this would seem to be an important benefit of the exemplar methodology. However, it can also be argued that what we glean by studying exemplars alone is sufficient to describe what these individuals are like with regards to their development in a particular area. If a characteristic or experience is evident among this sample, then it is evident. A comparison with a matched sample is not needed. Ultimately, the decision to use a comparison sample should be made in light on the claims that the researcher hopes to make. Without a comparison sample, researchers can claim that exemplary individuals possess certain characteristics and share particular experiences, but they cannot claim that these characteristics and experiences differentiate the exemplary individuals from more typical people.

Another complex issue with regards to the exemplar methodology has to do with the nature of exemplarity itself. How can researchers ascertain what constitutes exemplarity in any particular domain? Whose conception of exemplarity is most valid? Researchers conducting studies of moral exemplars regularly wrestle with what constitutes morality. Some studies of moral exemplars rely on lay conceptions (e.g. Walker \& Pitts 1998) and others on expert conceptions (e.g. Colby \& Damon 1992). Still others rely on behavioral manifestations of different aspects of morality (e.g. Walker \& Frimer 2007). The expert approach has the potential advantage of including a thoughtful and unbiased perspective on morality, but in some cases it ends up yielding a fairly narrow and often unrepresentative view of the construct (Matsuba \& Walker 2005). The lay perspective is likely to be broader and more representative, but it may also runs the risk of being diffuse and biased. The outcome approach can include engagement in particular acts (e.g. harboring Jews during the holocaust as a sign of altruism exemplarity; Oliner \& Oliner 1988) or winning special awards (e.g. winning national awards for care or bravery as signs of care or bravery exemplarity; Walker \& Frimer 2007). The outcome approach is likely to yield a more homogeneous sample, at least with regards to certain experiences around the construct of interest, but because it is narrower it may miss individuals who would meet the criteria, but who did not have the opportunity to engage in the exemplar qualifying acts. Ultimately, the best approach depends on the aims of the study, but regardless of the approach selected, it is important to consider the ways in which the chosen definition of exemplarity is likely to influence the study's findings.

Finally, it is important to consider how exemplar study findings can be generalized. Findings reveal the leading edge of development, but what does this tell us about more typical development of the construct? Correlational exemplar studies predict the experiences and characteristics that are likely to accompany the construct of interest, but we cannot claim that these experiences and characteristics cause exemplarity. Furthermore, it is important to bear in mind that while the exemplars are highly developed in one particular area, they are not necessarily highly developed in others, so it is important to bracket findings to the construct of interest. 
The discussion that follows outlines the steps involved in conducting an exemplar study. These conceptual issues, including the use of comparison studies, discernment of exemplarity, and generalization of findings, undergird and guide this discussion.

\section{Steps in conducting exemplar research}

To date, the exemplar methodology has largely been confined to studies or moral and ethical development. Researchers in this area, familiar with Colby \& Damon's (1992) influential study of moral exemplars, have applied the methodology in related studies of ethical development (e.g. care exemplars, Hart \& Fegley 1995; spiritual exemplars, King 2010; environmental exemplars, Pratt 2011; moral exemplars, Walker \& Frimer 2007). While the methodology is certainly useful in this area, it should be applied more broadly to studies of positive psychology. Use has been limited to a narrow sliver of the positive psychology space in large part because the methodology has rarely been written about. To encourage broader use of this methodology, guidelines for its implementation are needed. Therefore, following is a discussion of the steps involved in carrying out an effective exemplar study.

Successful use of the exemplar methodology requires special attention to sample selection. Individuals who exhibit signs of full or nearly full development in the area of interest are included in the study. To determine which individuals demonstrate complete or nearly complete development, most studies rely on carefully designed nomination criteria and thoughtfully selected nominators who use the nomination criteria to select appropriate exemplar participants.

\section{Nomination criteria}

One of the first steps to utilizing the exemplar methodology is to devise the criteria by which the exemplars are to be identified. Nomination criteria represent the standards used to qualify exemplars.

Researchers vary widely in terms of the rigor they apply to devising nomination criteria. One of the earliest and most influential exemplar studies employed a very thorough method in this regard. Colby \& Damon (1992) used an iterative process that relied on moral experts, including moral philosophers, theologians, ethicists, historians, and social scientists from different cultural and ethnic backgrounds, to develop their nomination criteria for moral exemplars. Interviews were conducted with the moral experts, in which they were presented with a preliminary list of criteria that the researchers believed offered a basis for identifying moral exemplars. Experts were encouraged to edit the list as they saw fit. Eventually, the following set of five criteria emerged:

1. Moral exemplars exhibit a sustained commitment to moral ideals or principles that include a generalized respect for humanity, or a sustained evidence of moral virtue,

2. a disposition to act in accord with their moral ideals or principles implying also a consistency between their actions and intentions and between the means and ends of their actions,

3. a willingness to risk their self-interest for the sake of their moral values,

4. a tendency to be inspiring to others and to move them to moral action, and

5. a sense of realistic humility about their own importance relative to the world at large, implying a relative lack of concern for their own egos (p. 29). 
Because the study sought to understand how morality developed and because people's ideas of what constitutes morality vary, it was important to have in place a systematic process for devising nomination criteria.

The expert approach to devising nomination criteria has been used in many exemplar studies. For example, a study of care exemplars by Hart \& Fegley (1995) asked community leader experts, including church and youth group leaders, to come together to develop criteria for care exemplars (Hart \& Fegley 1995). Their collaboration yielded the following criteria:

1. Youth care exemplars are involved in community, church, or youth group activities that benefit others,

2. have unusual and admirable family responsibilities,

3. exhibit a willingness to help those in need,

4. volunteer their time to help others,

5. display emotional and social maturity,

6. lead others,

7. practice open-mindedness about others,

8. demonstrate a willingness to look beyond the difficulties of living in an urban locale to a better future,

9. show compassion,

10. display a sense of humility about his/her aid to others, and/or

11. demonstrate a commitment to friends and family (p. 1350).

Relying on community leaders who oversaw youth engaged in care-oriented, volunteer work was appropriate since Hart and Fegley were interested in understanding the correlates of a highly developed sense of caring. A subsequent study of adolescent moral maturity (Reimer et al. 2009) used similar criteria to identify young moral exemplars, or "adolescent exemplars as paragons of moral maturity" (p. 380).

The outcome approach represents another effective technique for devising nomination criteria. Interested in identifying bravery and care exemplars, for example, Walker and Frimer (2007) used awardees who were nationally recognized for demonstrating exceptional levels of bravery or care. Similarly, Oliner and Oliner (1988) explored the highly developed altruistic personality by examining the lives of individuals who harbored Jews in Germany during the Holocaust. Individuals qualified as altruism exemplars ${ }^{\mathrm{a}}$ through their actions, which (1) were directed toward helping another, (2) involved high risk or sacrifice to the actor, (3) did not earn them any award, and (4) were voluntary (p. 6).

Still other studies have relied on existing literature and definitions of the construct to devise nomination criteria. For example, recent studies of youth purpose (Bronk 2008; Bronk 2011; Bronk 2012; Damon 2009) based their nomination criteria on the definition of purpose. Consistent with the definition, which states that a purpose in life is a stable and generalized intention to accomplish something that is at once meaningful to the individual and at the same time leads to productive engagement with some aspect of the world beyond the individual (Damon 2009; Damon et al. 2003), these studies relied on the following nomination criteria:

1. Youth purpose exemplars demonstrate enduring commitments to long-term aims,

2. are actively engaged in working toward their long-term aims and have plans for continuing to do so in the future, 
3. maintain long-term aims that are personally meaningful and central to their sense of self, and

4. demonstrate commitment to these aims in large part because pursuing them allows the youth to positively impact the broader world, including groups of people, causes, artistic endeavors, etc. (Bronk 2008; Bronk 2012, pp. 83-84).

Nomination criteria for a phenomenological study of moral commitment (MacRenato 1995) similarly relied on definitional components, including: (1) At least three years of sustained service to others outside of one's work life (2) and a demonstrated tendency to inspire others to engage in similar service.

The expert approach, the outcome approach, and the definitional approach represent three useful ways of devising nomination criteria. Exactly which approach is best is determined by the particular study aims, but effective exemplar studies share certain features, including a systematic and thoughtful approach to devising nomination criteria. This is key because who the exemplars are and which criteria are considered will significantly impact the study's findings. Nomination criteria should also draw useful boundaries around the construct; they need to be at once narrow enough to be descriptive of a particularly highly developed group of individuals but at the same time broad enough to capture the range of experiences and characteristics of highly developed individuals.

Second, researchers need to be mindful of the nomination criteria when reporting conclusions from their study to avoid reporting findings that were determined by the nomination criteria. For example, if the nomination criteria stipulate that civic exemplars demonstrate an other-oriented perspective, then experiences or characteristics that reveal a concern for the world beyond-the-self should not be reported as findings. In this way, hypotheses regarding likely study outcomes can help guide the creation of useful nomination criteria.

Third, because the aim of the criteria is to help nominators readily identify and agree upon potential exemplars, effective nomination criteria are concrete and quantifiable. For example, criteria that include visible behaviors or irrefutable phenomena are preferable to criteria that are vague or open to interpretation.

Additionally, some studies include demographic criteria. Researchers may choose to select a sample balanced for gender, age, ethnicity, income, or by other demographic variables.

\section{Nominating exemplars}

Once nomination criteria have been established, a process needs to be instituted for using the criteria to select the exemplars. Generally a small group of individuals is invited to serve as nominators. In some cases, the same individuals who created the criteria serve as nominators (Colby \& Damon 1992; Hart \& Fegley 1995). This expert approach has the benefit of ensuring that nominators fully understand and can apply the nomination criteria. However, the nominators may be too close to the project and may give extra weight to certain criteria. Of course, new nominators run this same risk, but there is less chance they would feel as invested in the criteria, and therefore would be more likely to recommend individuals who meet the stated criteria. Alternatively, using 
the same individuals to create the nomination criteria and select exemplars could be advantageous, since these individuals may struggle to verbalize some of their criteria, but may apply them in selecting relevant individuals.

Many exemplar studies rely on a new set of nominators to avoid having nominators who are too close to the project or because there are no outside individuals involved in the process of developing the nomination criteria. For instance, in the earlier referenced study of youth purpose, the nomination criteria were largely based on the definition of purpose, and youth practitioners, including coaches, teachers, youth ministers, etc., were called upon to nominate youth who met the criteria (Damon 2009; Bronk 2008; Bronk 2011; Bronk 2012).

Other exemplar studies use practitioners as nominators. These studies may invite leaders of local human service organizations such as shelters, orphanages, schools, and outreach programs to nominate altruism exemplars (Mastain 2008), leaders of community-service organizations such as The Ronald McDonald House, AIDs organizations, and churches to nominate moral exemplars (Matsuba \& Walker 2005), or youth workers to nominate care exemplars (Reimer \& Wade-Stein 2004) based on their nomination criteria.

Still other exemplar studies employ laypeople as nominators. For example, Walker \& Matsuba (2005) asked members of social organizations to nominate young adults within their organizations who had demonstrated "extraordinary moral commitments" (p. 276). An interpretation of what constituted an extraordinary moral commitment was left up to the lay nominator. The decision to use relatively vague nomination criteria and to include lay individuals as nominators was an intentional one; the research team was concerned that expert nominators and more prescriptive nomination criteria would reflect too narrow a conception of morality, and the team sought to identify exemplars who reflected a "folk" conception of moral excellence (Walker \& Matsuba 2005, p. 281).

\section{Comparison samples}

In addition to devising appropriate nomination criteria and selecting suitable nominators, effective exemplar studies often employ comparison samples (e.g. Bronk 2008; Bronk 2011; Frimer \& Walker 2011; Hart \& Fegley 1995; Matsuba \& Walker 2004; Oliner \& Oliner 1988; Reimer et al. 2009; Walker \& Frimer 2007). Ideally, comparison samples should be demographically matched with the exemplars to isolate the variable of interest. While particular demographics of interest may vary based on the nature of the study, age, gender, ethnicity, social status, educational level, and hometown are useful variables to consider for matching purposes. Without a comparison sample, it is difficult, if not impossible, to make strong claims about unique attributes or experiences associated with exemplarity. However, when researchers do not seek to distinguish exemplar from more typical cases, comparison samples are not needed (e.g. Bronk 2012; Colby \& Damon 1992; Midlarsky et al. 2005).

\section{Data analysis}

An exemplar study is determined by its participants rather than by its data analysis strategy. As such, data analysis options vary. In some exemplar studies, researchers administer surveys to relatively large numbers of exemplar participants and data analysis is quantitative in nature (Matsuba \& Walker 2004; Walker \& Frimer 2007). In other cases, 
fewer participants are included and data analysis is qualitative in nature (Bronk 2008; Bronk 2011; Bronk 2012; Colby \& Damon 1992; Damon 2009; Hart \& Fegley 1995; King 2010; Matsuba \& Walker 2005; Walker \& Frimer 2007). In line with historical uses of the exemplar methodology (e.g. Colby \& Damon 1992), qualitative exemplar studies rely on semi-structured, case study style interview protocols that are used for gathering both nomothetic and idiographic data. In still other exemplar studies, mixed methods data analysis approaches are employed (Matsuba \& Walker 2004; Walker \& Frimer 2007).

Most exemplar research is qualitative in nature. One reason may have to do with the history of this kind of research; early exemplar studies were qualitative in nature (Colby \& Damon 1992; Hart \& Fegley 1995). A second reason likely has to do with the difficulty of securing a sample that is at once exemplary and at the same time large enough for statistical analyses. Typically quantitative exemplar studies are required to lower the threshold of exemplarity to garner a sufficient sample size, but the benefits of doing so may be great enough to warrant such a decision.

\section{Generalizing findings}

Examining exemplars' lives allows us to understand and even predict the developmental trajectory of the construct of interest. Exemplars characterize the leading edge of development, and their lives can be generalized in a developmental sense, but not from a conduct perspective. For example, when more typical individuals manage to act in an altruistic manner every now and again, they do so in the same way that altruism exemplars, including those who dedicated their lives to saving Jews from the holocaust, managed to do consistently. However, while the process of developing altruism may be the similar for exemplars and typical individuals, the conduct of exemplars is likely to differ from the conduct of typical individuals; there are of course many ways of acting altruistically.

\section{Conclusion}

Conducting exemplar research can add a great deal to our understanding of positive developmental phenomena. It provides a picture of complete or nearly complete development, and adding this understanding to research on deficient and typical growth allows us to examine the full spectrum of development; accordingly, the exemplar methodology is a critical research tool (Damon \& Colby in press). However, as spelled out here, the methodology needs to be rigorously and thoughtfully applied.

As discussed in the introduction, Maslow (1971) was one of the earliest psychologists to conduct exemplar research. Interested in understanding what constituted selfactualization, Maslow identified twenty-one highly self-actualized individuals and studied them. In spite of the influential results generated by this investigation, some have argued that Maslow's use of the exemplar methodology was not scientific in nature (Sommers \& Satel 2006). Had Maslow followed the guidelines set forth by existing exemplar studies, he could have avoided these critiques.

First, Maslow identified individuals he believed were self-actualized. He then read essays, books, and other papers written by the exemplars of self-actualization and tried to infer personality characteristics from the written documents. Not surprisingly, he has been roundly criticized for his vague and potentially biased process of exemplar identification (Sommers \& Satel 2006). Including a rigorously selected panel of 
nominators and including clearly stated nomination criteria would have clarified the basis on which these individuals qualified as exemplary self-actualizers and would have minimized the potential influence of personal bias.

Second, not only did Maslow draw conclusions about the characteristics of selfactualizers based on his sample, but he also drew conclusions regarding the nature of self-actualization. Here Maslow committed one of the common errors in exemplar studies, and one that is warned against in this paper. Maslow drew conclusions based on his inclusion criteria, and doing so is tautological in nature. Had he clearly stated what his nomination criteria were, it would have been easier to avoid drawing conclusions that were predetermined by them.

Finally, it is possible that individuals other than self-actualizers possess the same characteristics as the exemplars. Because Maslow was interested not only in understanding more about the concept of self-actualization, but also in differentiating self-actualizers from more typical individuals, a comparison sample would have strengthened his study. Comparison samples are called for when the researcher intends to draw distinctions between exemplary and more typical individuals.

In sum, Maslow acknowledged that his methodology was lacking, and he tried to draw tentative conclusions regarding his findings. His hope was that future researchers would extend his ideas and investigate them more rigorously, but by following the aforementioned methodological guidelines, he could have avoided these shortcomings altogether. The fact that his findings are still so highly regarded and regularly debated underscores the interesting and important findings that exemplar research- even flawed exemplar research- can uncover.

Given that the exemplar methodology has become an increasingly popular means of investigating ethical constructs such as morality, spirituality, activism, care, bravery, environmental activism, and purpose in life, the time has come to outline the parameters of and draw boundaries around the reaches of this useful methodology. Additionally, the present article has attempted to outline key components of effective exemplar methodologies, including the importance of devising thoughtful nomination criteria, identifying appropriate nominators, using matched comparison samples, analyzing data, and generalizing findings. Clearer guidelines regarding how to carry out an exemplar study should allow a broader range of researchers to make use of this approach. Doing so will be reveal a fuller understanding not only of what is, but also of what is possible in terms of human development.

\section{Endnotes}

a "Altruism exemplars" is the author's term, not Oliner and Oliner's, but given their research methodology, it seems applicable.

Competing interests

The author declares she has no competing interests.

Received: 18 June 2012 Accepted: 6 October 2012

Published: 16 October 2012

References

Aristotle. (1962). Nicomachean Ethics (Translated by Martin Ostwald). Indianapolis: The Library of Liberal Arts,

Benson, P. L., Scales, P. C., Hamilton, S. F., \& Sesma, A., Jr. (2006). Positive youth development: Theory, research and applications. In W. Damon \& R. M. Lerner (Eds.), Handbook of child psychology, 6th ed., Vol. 1, Theoretical models of human development (pp. 894-941). New York: John Wiley. 
Bronk, K. C. (2008). Humility among purpose exemplars. Journal of Research in Character Education, 6(1), 35-51. Bronk, K. C. (2011). The role of purpose in identity formation. A special issue on Instructing for and Supporting Youth Purpose. New Directions in Youth Development, 132, 31-44.

Bronk, K. C. (2012). A grounded theory of the development of youth purpose. Journal of Adolescent Research, 27, 78-109. http://dx.doi.org/10.1177/0743558411412958.

Bundick, M. J., Yeager, D. S., King, P. E., \& Damon, W. (2010). Thriving across the lifespan. In R. M. Lerner, M. E. Lamb, A. M. Freund, \& W. F. Overton (Eds.), Handbook of life- span development, Vol. 1: Cognition, Biology and Methods (pp. 882-923). Hoboken, NJ: John Wiley \& Sons.

Colby, A., \& Damon, W. (1992). Some do care. New York: The Free Press.

Damon, W. (2004). What is positive youth development? The ANNALS of the American Academy of Political and Social Science, 591, 13-24.

Damon, W. (2009). The path to purpose: How young people find their calling in life. New York: Free Press.

Damon, W., \& Colby, A. (in press). Why a true account of human development requires exemplar research. In K. Matsuba, P. E. King, \& K. C. Bronk (Eds.), Exemplar methods and research: Quantitative and qualitative strategies for investigation. New Directions in Child and Adolescent Development.

Damon, W., Menon, J., \& Bronk, K. C. (2003). The development of purpose during adolescence. Applied Developmental Science, 7(3), 119-128.

Frimer, J., \& Walker, L. (2011). Enlightened self-interest in the lives of moral heroes (Paper presented at the biennial meeting of the Society for Research in Child Development. Montreal, Canada).

Hart, D., \& Fegley, S. (1995). Prosocial behavior and caring in adolescence: Relations to self-understanding and social judgment. Child Development, 66(5), 1346-1359.

King, P. (2010). Spiritual exemplars from around the world: An exploratory study of spiritual development among adolescents (Paper presented at the 2010 biennial meeting of the Society for Research on Adolescence, Philadelphia, PA).

Lerner, R. M., Dowling, E. M., \& Anderson, P. M. (2003). Positive youth development: Thriving as a basis of personhood and civil society. Applied Developmental Science, 7, 172-180.

MacRenato, S. W. (1995). Experiences of moral commitment: A phenomenoligcal study. San Diego, CA: University of San Diego.

Maslow, A. (1971). The farther reaches of human nature. New York: The Viking Press.

Mastain, L. (2008). A phenomenological investigation of altruism as experienced by moral exemplars. Journal of Phenomenology, 38, 62-99.

Matsuba, M. K., \& Walker, L. (2004). Extraordinary moral commitment: Young adults involved in social organizations. Journal of Personality, 72(2), 413-436.

Matsuba, M. K., \& Walker, L. (2005). Young adult moral exemplars: The making of self through stories. Journal of Research on Adolescence, 15(3), 275-297.

Matsuba, M. K., King, P. E., \& Bronk, K. C. (Eds.). (in press). Exemplar methods and research: Qualitative and quantitative strategies for investigation (New Directions in Child and Adolescent Development).

Midlarsky, E., Jones, S. F., \& Corley, R. P. (2005). Personality correlates of heroic rescue during the Holocaust. Journal of Personality, 73, 907-934.

Oliner, S. P., \& Oliner, M. P. (1988). The altruistic personality: Rescuers of Jews in Nazi Europe: What Led Ordinary Men and Women to Risk their Lives on Behalf of Others? New York: The Free Press.

Pratt, M. (2011). Environmental memories of youth and adults: Identity and exemplar status predict story recall and level of detail (Paper presented at the biennial meeting of the Society for Research on Child Development. Montreal, Canada).

Reimer, K. (2003). Committed to caring: Transformation in adolescent moral identity. Applied Developmental Science, 7(3), 129-137.

Reimer, K., \& Wade-Stein, D. (2004). Moral identity in adolescence: Self and other in semantic space. Identity: An International Journal of Theory and Research, 4(3), 229-249.

Reimer, K., Goudelock, B., \& Walker, L. (2009). Developing conceptions of moral maturity: Traits and identity in adolescent personality. Journal of Positive Psychology, 4, 372-388.

Seligman, M. E. P. (2011). Flourish: A visionary new understanding of happiness and well-being. New York: Free Press.

Seligman, M., \& Csikszentmihalyi, M. (2000). Positive psychology: An introduction. American Psychologist, 55, 5-14.

Sheldon, K. M., \& King, L. A. (2001). Why positive psychology is necessary. American Psychologist, 56, 216-217.

Sommers, C. H., \& Satel, S. (2006). One Nation Under Therapy: How the Helping Culture is Eroding Self-reliance. New York: McMillian.

Walker, L. J., \& Frimer, J. A. (2007). Moral personality of brave and caring exemplars. Journal of Personality and Social Psychology, 93(5), 845-860

Walker, L., \& Matsuba, M. K. (2005). Young adult moral exemplars: The making of self through stories. Journal of Research on Adolescence, 15(3), 275-297.

Walker, L. J., \& Pitts, R. C. (1998). Naturalistic conceptions of moral maturity. Developmental Psychology, 34, 403-419.

doi:10.1186/2211-1522-2-5

Cite this article as: Bronk: The exemplar methodology: An approach to studying the leading edge of

development. Psychology of Well-Being: Theory, Research and Practice 2012 2:5. 